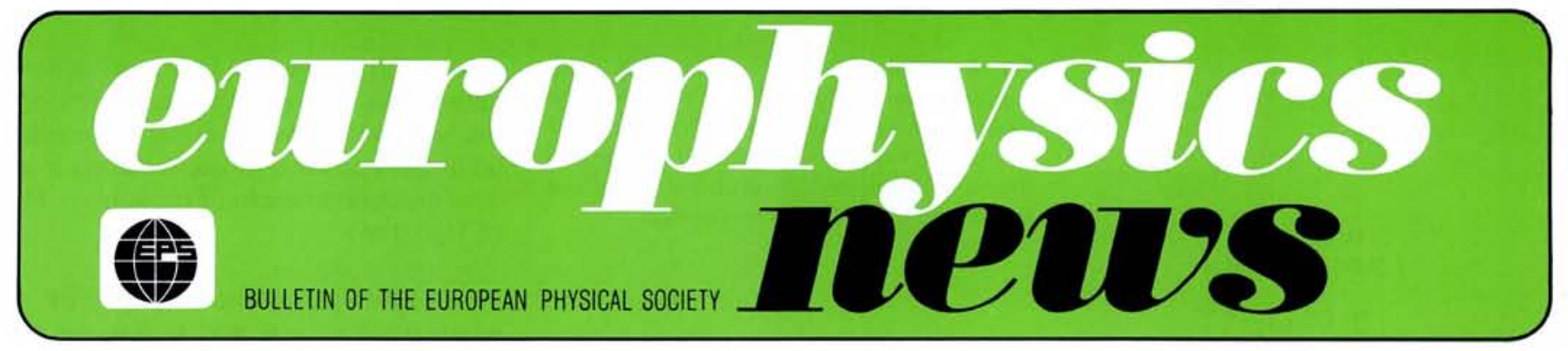

\title{
Neutron Spin Echo Spectroscopy
}

\author{
F. Mezei, Grenoble / Budapest * \\ (Institut-Laue-Langevin / Central Research Institute for Physics)
}

Inelastic neutron scattering is potentially the ultimate tool for the investigation of atomic and magnetic dynamics on the microscopic scale in condensed matter. This is because of the unique feature that both the wavelength and the energy of thermal neutron radiation fall within the range relevant to the dynamics of common solids and liquids viz. $1-10 \AA$ and $1-100 \mathrm{meV} \cong 0.25-25 \mathrm{THz}$. Neutron scattering is the only microscopic probe able to provide the whole picture in space and time although there are a number of other methods, which yield partial information. For example, X-ray scattering is very powerful in the determination of atomic structures, but since $X$-ray quanta have energies in the $10 \mathrm{keV}$ range, it is not practical (at least so far) to observe changes on the $\mathrm{meV}$ or $\mu \mathrm{eV}$ scale associated with atomic motions. To a very rough approximation, the energy changes of the scattered radiation can be looked upon as Doppler shifts caused by the motions of the scattering atoms. In contrast, with light scattering one can observe easily any energy change that might occur, but the basic wavelength of several $1000 \AA$ restricts the space domain studied to one of similar size, i.e. to practically macroscopic regions. Or, to take an example at the other extreme, nuclear magnetic reso-

\footnotetext{
* Present Address:

Hahn-Meitner-Institut and Technical University, Berlin (West)
}

nance (NMR) allows us to study local fluctuations at nuclear sites at an adequate rate, but does not allow us to make direct observation of correlations between neighbouring atomic sites.

Further very useful features of neutron radiation are its interaction with the magnetic moments in the sample via the neutron spin and its large penetration into many materials. This latter is made use of in industrial applications like neutron radiography and testing for phase homogeneity in welds by neutron diffraction.

Inevitably, such outstanding advantages cannot occur in real life without drawbacks! For neutrons there are two: neutron sources are expensive and even the best available beam fluxes are small in absolute terms (i.e. compared with the number of atoms in a sample). Thus, while in an NMR experiment we typically have $10^{20}$ nuclear spins to act on, or a laser can provide $10^{20}$ light quanta within reasonable time, the highest flux neutron scattering instruments barely provide $10^{13}$ neutrons over a day. Consequently only relatively big samples and/or strong scattering effects can be studied with neutron scattering and the statistical accuracy of the results is always limited. As a rule of thumb, neutron scattering investigation, giving a detailed, model-independent space-time picture, is indispensable if we are not absolutely sure of the nature of a particular phenomenon, whereas systematic studies on a large number of similar systems, including small samples, is better done by methods mobilizing a larger number of quanta.

For a (neutron) experiment to be productive it is not sufficient to have the right kind of probe, one has also to be able to extract enough information. This is the problem of experimental resolution (which is, e.g. the essential reason why we cannot use $\mathrm{X}$-ray scattering for the study of, say, phonons). Conventional neutron scattering methods allow us to determine the energy changes of the neutron radiation in the scattering process with a typical best resolution of $1 \%$. This limits the range of frequencies which can be studied to about $10 \mathrm{GHz}$ $20 \mathrm{THz}$. (Epithermal neutron beams which are becoming available with proper intensities at the so-called "spallation sources", extend this range to maybe $500 \mathrm{THz}$.)

\begin{tabular}{|lr|}
\hline Contents & \\
Neutron Spin Echo Spectroscopy & 1 \\
New Members of EPS & 4 \\
Neutral Injection Heating in & \\
$\quad$ Fusion Devices & 5 \\
Nuclear Methods in Condensed & \\
$\quad$ Matter Physics Studies & 9 \\
$\begin{array}{l}\text { Hewlett-Packard Prize } \\
\text { Surface Studies of MBE-Grown }\end{array}$ & 16 \\
$\quad \begin{array}{l}\text { Semiconductor Films } \\
\text { Council Decisions }\end{array}$ & 12 \\
$\begin{array}{l}\text { Changes to EPS Constitution } \\
\text { and By-Laws }\end{array}$ & 15 \\
IOM Delegate to Council & 16 \\
\hline
\end{tabular}




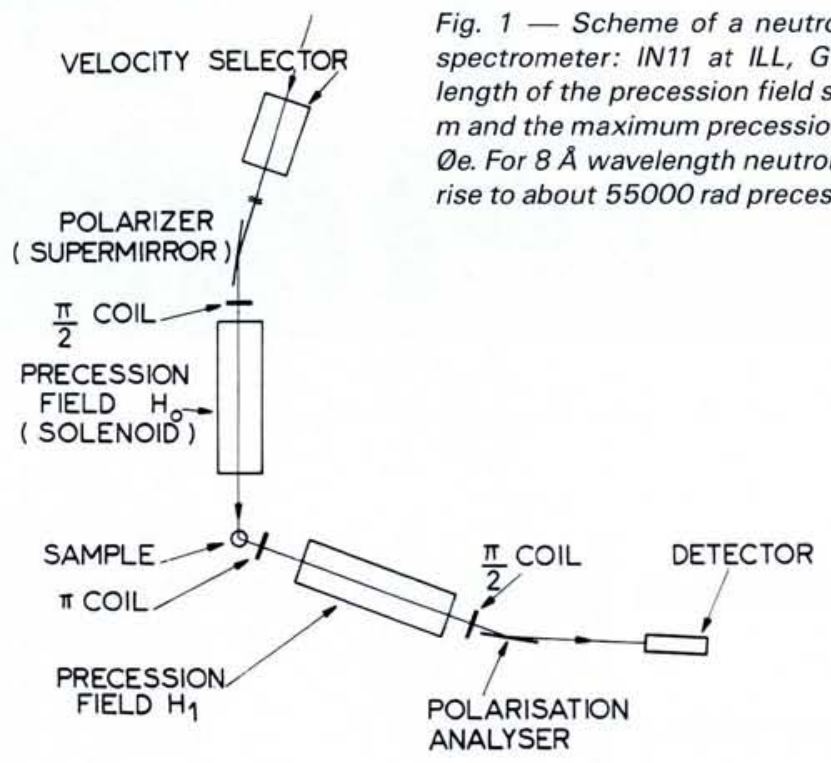

In order to study slower phenomena, the energy resolution had to be improved well beyond the $1 \%$ level. The main difficulty in doing this was not really technical but fundamental: the problem of low beam intensity. The production of a highly monochromatic beam means selecting out a tiny portion from the originally Maxwellian neutron energy distribution. Since low beam intensities are the main limitation in neutron scattering from the outset, the direct path to higher resolution is basically limited by the flux alone. The so-called "backscattering" method, in which $0.01-0.1 \%$ monochromatic beams are used, follows this conventional path. The price one has to pay for the gain in energy (time) resolution $(30-100 \mathrm{MHz}$ lower limit) is that in order to recover some of the lost intensity, the momentum (space) resolution has to be relaxed. Logically, the method has proved to be extremely successful, primarily in the study of non-dispersive (wavenumber independent) phenomena, such as the tunnelling motion of protons and other radicals between various local equilibrium positions within the elementary cell of a crystal.

To overcome the fundamental intensity barrier to higher resolution a radically new approach was needed: the resolution had to be decoupled from the monochromatisation. This apparent contradiction is solved in the neutron spin echo (NSE) method ${ }^{1}$ ). The basic idea is that instead of monochromatizing the beam impinging on the sample, we make each neutron remember its initial velocity. In order to do this, we use the natural individual clock each neutron possesses: its spin. In effect, the Larmor spin precession frequency $\omega_{\llcorner}$in an external ma- gnetic field $H$ conveniently lends itself to its use as a time base:

$$
\omega_{\mathrm{L}}=\gamma_{\mathrm{L}} \mathrm{H}
$$

where the constant $\gamma_{L}=2.916 \mathrm{kHz} / \mathrm{Oe}$.

If a neutron with a velocity $v$ crosses a magnetic field of strength $H$ and length $\ell$, the total Larmor precession angle $\varphi$ will be a measure of its velocity:

$$
\varphi=\gamma_{L} H \ell / v
$$

In writing down this equation we implicitly assume that the neutron can be considered as a classical particle, i.e. it is pointlike and thus has a well defined trajectory and velocity, while its spin corresponds to a classical vector and performs precessions in a field in the classical mechanical sense, like a top. This is certainly at variance with the "popular" picture, of the spin of a spin $1 / 2$ particle being able to occupy only discrete "up" and "down" states. Such a picture is, of course, an incorrect over-simplification, but it was the reason nevertheless why for a long time, little effort was made to explore the full vectorial character of spin polarization in particle beam experiments. Rigorous quantum mechanical analysis shows ${ }^{3}$ ), that in magnetic fields where the gradients are not too strong (in the absence of the SternGerlach quantum effect) the neutron spin motion can be treated classically, i.e. by considering the Larmor precessions governed by the classical equation:

$$
d \mathbf{S} / d t=\gamma_{L}[\mathbf{S} \times \mathbf{H}]
$$

where $\mathbf{S}$ is the spin vector.

In a neutron spin echo spectrometer (Fig. 1) a first "precession" field is used to allow each neutron to label its own initial velocity $v_{0}$ by performing a precession of $\Phi_{0}$, and after scattering on the sample a second precession field is used to measure the final velocity $v_{1}$ via the precession angle $\Phi_{1}$. The comparison between $\varphi_{\mathrm{o}}$ and $\varphi_{1}$ is made by making the two precessions to occur (effectively) in the opposite sense, resulting in a total precession angle (if $H_{0}=H_{1}=H$, cf. Fig. 1) of:

$$
\begin{aligned}
\varphi & =\varphi_{\mathrm{o}}-\varphi_{1} \\
& =\gamma_{\mathrm{L}} H \ell\left(1 / v_{\mathrm{o}}-1 / v_{\mathrm{I}}\right) \cong \gamma_{\mathrm{L}} H \ell v_{\mathrm{o}}{ }^{-2} \delta v
\end{aligned}
$$

where $\delta v=v_{1}-v_{0}$ and we assume $\delta v$ $<<v_{0}$. Remembering that the neutron energy is $1 / 2 m v^{2}$, we see that $\varphi$ is just a measure of the neutron energy change in the scattering process, $\hbar \omega$, which is what interests us:

$$
\Phi=\left(\gamma_{L} H \ell / m v_{0}{ }^{3}\right) \hbar \omega=t \omega
$$

if $v_{0}$ is rather well defined (in practice beams with about $\pm 10 \%$ variation of $v_{0}$ are used) the proportionality constant $t=\gamma_{L} H \ell / m v_{0}^{3}$ is also.

The important thing is that the observable quantity $\varphi$ is directly related to the change of the neutron energy, and we do not have to proceed by the determination of the initial and final neutron energies in two separate steps. Therefore $\hbar \omega$ can be determined independently of the scatter of the initial and final neutron energies, and, for the first time, the energy resolution becomes independent of the monochromatization of the beam. This means that we have managed to side-step the normal reciprocal relation between resolution and beam intensity.

The fundamental practical point in NSE is how to produce and analyse Larmor precessions. This can be done surprisingly easily with the help of a simple flat coil (Fig. 2), whose introduction in 1972 at the Central Research Institute for Physics in Budapest was actually the starting point of NSE ${ }^{1}$ ). If neutrons enter the coil with spin $\vec{S}$ parallel to the external field $\vec{H}$, inside the coil they will start to process around the field $\vec{H}^{\prime}$ which is the sum of the external field $\vec{H}$ and the field $\vec{H}_{\mathrm{c}}$ produced by the coil. If, as shown, the neutrons leave the coil after half a precession around $\vec{H}^{\prime}$ which
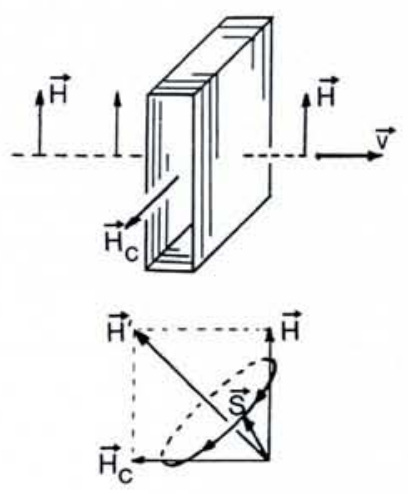

Fig. 2 - The key element of NSE spectroscopy: the spin flip coil (typically activated by 1-3A DC current) and an example of spin turn by Larmor precession inside the coil. 


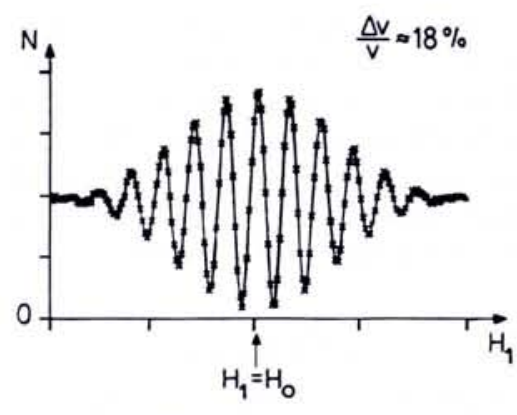

Fig. 3 - NSE signal showing the echo effect as measured with an $18 \%$ FWHM velocity-selected beam. The dephasing and decrease of the precessing polarization on both sides of the $H_{o}=H_{1}$ echo condition reflect this $18 \%$ spread of neutron velocities.

is bisecting the directions of $\vec{H}$ and $\vec{H}_{c^{\prime}}$ the effect of the coil is to turn $\vec{S}$ from the direction of $\vec{H}$ into that of $\vec{H}_{c}$. This is how the Larmor precessions can be initiated by turning through $90^{\circ}$ the neutron spins, originally parallel to the field (Fig. 2 ). The inverse procedure, turning a component of the precessing polarisation (i.e. perpendicular to the field) into the field direction and using a conventional spin analyser, allows us to observe Larmor precessions. It turns out that the fields required to achieve such spin turns are rather modest: e.g. with $1000 \mathrm{~m} / \mathrm{s}$ ( $4 \AA$ wavelength) neutrons and a $1 \mathrm{~cm}$ thick coil, $H$ and $H_{c}$ have to be of $12.1 \emptyset \mathrm{e}$ in order to achieve a $90^{\circ}$ spin turn (cf eq. (2)). Moreover, neutron radiation will traverse the single layer of Al wire windings that make up the coil without appreciable intensity loss.

The analysis of the precessing polarisation in the beam does not mean in practice the direct measurement of the angle $\varphi$, but the observation of one component (say $x$ ) of the polarization $P$. If $\varphi$ is measured with respect to the $x$ axis, we have

$$
P_{x}=\langle\cos \varphi\rangle
$$

where the bracket stands for the average over all neutrons in the beam. Thus, in view of eq. (3), in a NSE experiment we determine

$P_{\mathrm{NSE}}=\langle\cos \varphi\rangle \cong \int S(\omega) \cos (t \omega) d \omega$

where $S(\omega)$ is the probability distribution of $\hbar \omega$ neutron energy changes in the scattering. It is known from the fundamentals of neutron scattering that $S(\omega)$ is just the Fourier transform of the correlation function $S(t)$ describing the atomic motions in real time. Since eq. (4) implies a backward Fourier transformation, it is $S(t)$ that we measure directly. Thus e.g. in a simple relaxation decay process we have

$$
P_{\mathrm{NSE}}=S(t)=\exp (-\Gamma t)
$$

where $\Gamma$ is the relaxation rate. The direct exploration of the time domain is sometimes a very useful additional feature of NSE. Note that in an NSE experiment $P_{\text {NSE }}$ is determined as a function of $t$, the latter being varied most simply by changing the precession field $H$, (cf eq. (3)).

Evidently the name given to the method has little to do with the above considerations, rather is it justified technically. Fig. 3 shows what we actually observe measuring the $x$ component of the precessing polarization after the second precession field $H_{1}$ as a function of that field ${ }^{1}$ ). As we have seen, at $H_{0}=$ $H_{1}, \varphi$ will be the same, i.e. zero, for all neutrons with $v_{1}=v_{0}$. This is not the case when $H_{0} \neq H_{1}$, and the distribution of $v_{0}\left(\right.$ or $v_{1}$ ) introduces a distribution of precession angles $\varphi$ which ultimately makes $P_{x}$ average to zero. Therefore the "echo signal" in Fig. 3 can only be observed around $H_{0}=H_{1}$ (if $v_{0} \cong v_{1}$ ). It is interesting to note that this echo signal is just the image of how the neutron waves in the beam would look if we described them by quantum mechanical (coherent) wave-packets as opposed to a distribution of classical velocities $v$. The two approaches lead to exactly the same results, of course ${ }^{3}$ ).

Since the IN11 NSE spectrometer (built by the author in collaboration with Paul Dagleish and John Hayter) went into operation in 1978 at the ILL, many successful and significant experiments have been performed on the dynamics of polymers and biopolymers in solutions, on atomic and molecular diffusion in liquids and solids, on critical fluctuations in structural and magnetic phase transitions, on the nature of the spin glass transition, on the decay of elementary excitations in superfluid helium, on soliton dynamics, etc. It is not our purpose here to consider in depth any of these results (an early summary can be found in Ref. 2). To illustrate the performance of the method only we shall look at just two examples instead.

Fig. 4 shows the relaxation rate $\Gamma$ of correlations in a solution of pig immunoglobulin G (IgG) molecules as a function of the wavenumber $q^{4}$ ). We expect $\Gamma$ to reflect diffusive motion: in time $t$ the molecules diffuse away on the average by a distance $r=\sqrt{ }(D t)$ (where $D$ is the diffusion constant) and thus the lifetime of $r=1 / q$ wavelength correlations is

$$
\Gamma^{-1}=r^{2} / D \text { or } \Gamma=D q^{2},
$$

which is the well known diffusion equation. The results in the figure show this relation in a $q$ and $\Gamma$ domain, in practice only accessible by NSE. The diffusion constant $D$ corresponding to the straight line in the figure was found to be $40 \%$ higher than that relevant to $q=0$ as

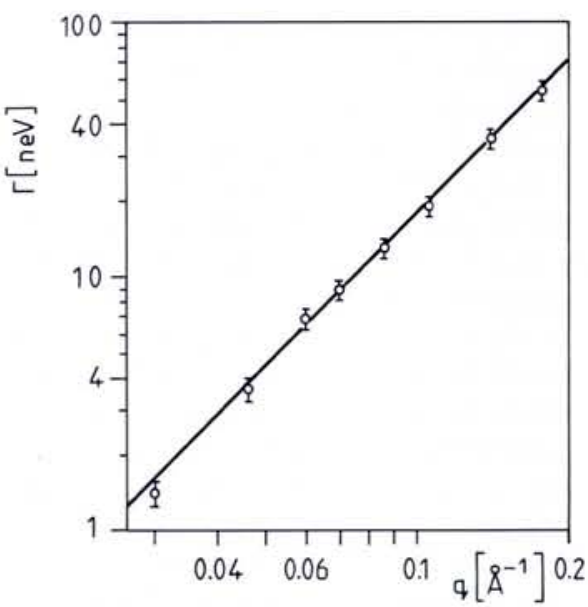

Fig. 4 - The relaxation rate measured in pig immunoglobulin $\mathrm{G}-\mathrm{D}_{2} \mathrm{O}$ solution of 7.33 $w t . \%$ concentration 4). The line corresponds to the $D q^{2}$ law with a diffusion constant $D=2.74 \times 10^{-7} \mathrm{~cm}^{2} \mathrm{~s}^{-1}$.

determined by sedimentation. Observing that the $q$ range of the NSE experiment corresponds to the size of the constituant subunits of the $\operatorname{lgG}$ molecule, this difference can be interpreted as evidence for intramolecular motion: on a length scale smaller than the size of the molecule, the subunits move faster than the molecule as a whole. On the purely technical side, on the other hand, note that the neutron energy changes (corresponding to the $\Gamma$ values in the figure) are on the neV $\left(10^{-9} \mathrm{eV}\right)$ scale compared with the incoming neutron energies that

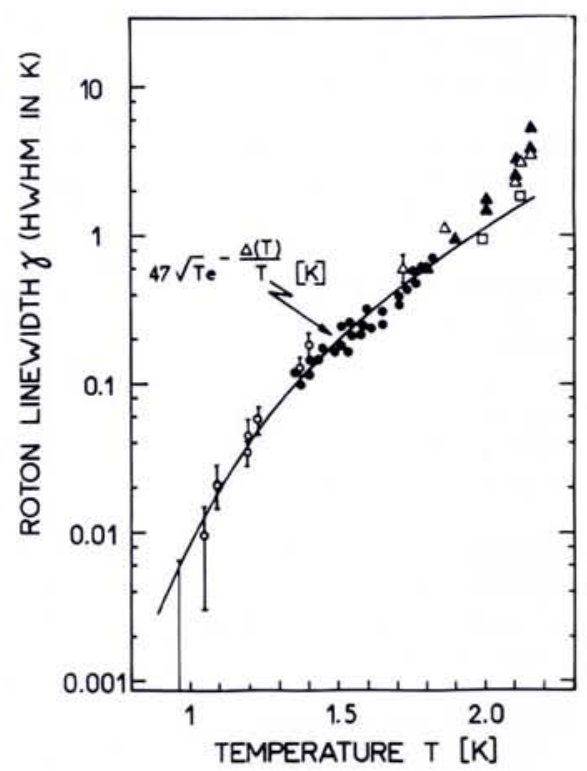

Fig. 5 - Decay rate of the roton excitation in superfluid ${ }^{4} \mathrm{He}$ at various temperatures ${ }^{5}$ ). The NSE results (open circles) are compared with indirect light scattering data (dots) and conventional neutron scattering results (other symbols) resolution limited to line widths above $0.5 \mathrm{~K}$. The line represents the theoretical prediction for temperature well below the $\lambda$ point $(2.17 \mathrm{~K})$. 
are around $1.2 \times 10^{-3} \mathrm{eV}$ with a scatter of $\pm 10 \%$. This is a fine illustration of the independence of energy resolution and beam monochromatization, which is the essence of NSE. These results represent the present absolute resolution limits in inelastic neutron scattering $\left(10^{-9} \mathrm{eV}\right.$ corresponds to $0.25 \mathrm{MHz}$ ).

The other example (Fig. 5) shows a rather more elaborate use of NSE: the measurement of the lifetime of roton excitation in superfluid $\left.{ }^{4} \mathrm{He}^{5}\right)$. In this case the deviation of the neutron energy change from a well defined value,

$\hbar \omega_{\mathrm{o}}=8.61 \mathrm{~K}=0.742 \mathrm{meV}$

(the minimum roton energy) is observed by the NSE difference method. This is done by using a special $H_{0} / H_{1}$ precession field ratio. NSE in this case provides a resolution nearly two orders of magnitude better than classical neutron scattering methods. The existence of light scattering results for comparison (full circles) is also quite exceptional: it is the appearance of two-roton bound states with total $q \cong 0$ that makes the high wave number $\left(q=1.92 \AA^{-1}\right)$ roton excitation accessible to investigation.

These examples taken from amongst many demonstrate that by the application of a new approach, the domain of applicability of inelastic neutron scattering, a fundamental tool in condensed matter research, could be substantially extended. It is worth noting that the new method is based on ideas and techniques which were originally developed at one of the smallest neutron research facilities in Europe, namely that in Budapest, and which were then completed and fully exploited at the most powerful facility in Grenoble. This is certainly a rather fortunate, but nevertheless significant example of fruitful collaboration in Europe.

\section{REFERENCES}

1. Mezei F., Z. Physik 255 (1972) 146. 2. Neutron Spin Echo, ed. F. Mezei (Springer Verlag, Heidelberg) 1980.

3. See Mezei F., J. de Physique 45 (1984) C3-223, and references therein.

4. Alpert Y., Cser L., Farago B., Franek F., Mezei F. and Ostanevich Y.M., Symposium on Neutron Scattering, Berlin (West), 6-8 August 1984

5. Mezei F., Phys. Rev. Lett. 44 (1980) 1602.

\section{New Members \\ of the European Physical Society}

\section{CATEGORY 4a)}

D. Blachon, Ste-Foy-les-Lyon, F

S.I.A. Garpman, Röstanga, S

A. Lisini, Trieste, I

C. Morgan, Frankfurt/Main, D

R. Polloni, Venezia, I

\section{CATEGORY 4c}

Austrian Physical Society

H. Winter, Vienna

\section{Danish Physical Society \\ J. Bohr, Roskilde \\ V.O. Jensen, Roskilde \\ J. Onsgaard, Odense}

Eötvös Lorand Physical Society P. Mogyorosi, Szeged

J. Szep, Budapest

Finnish Physical Society

M. Brenner, Abo

I. Liede, Vantaa

M. Kajanto, Espoo

S. Malkamäki, Rusko

T.T. Tuuva, Helsinki

R. Vatanen, Punkalaidun

French Physical Society

P. Chavel, Orsay

Ch. Hirlimann, Asnières s/Seine

P. Mein, Meudon

\section{German Physical Society}

A. Bradshaw, Berlin (W)

J. Debrus, Bad Honnef

U. Eckern, Karlsruhe

K. Grob, Ludwigsburg

H.-G. Kilian, Ulm

F. Koch, Munich

M. Tonutti,

Aachen-Kornelimuenster

Hellenic Physical Society

D. Bonatsos, Philadelphia, USA
The Institute of Physics

G.A. Bell, Maidenhead

A.F. Khater, Hadath Beirut, LE

J. Lang, Abingdon

J.B. Pendry, London

A. Riera, Madrid, SP

R.A. Stradling, London

J.D. Sutton, Rijswijk, NL

M.J. Tuck, Denbigh

Italian Physical Society

U. Balucani, Florence

V. Bellini, Catania

M. Bertero, Genova

V. Bortolani, Modena

F. Chiuderi-Drago, Meudon, F

V. Flaminio, Ghezzano

B. Ghidini, Bari

U. Giorgiani, Messina

R. Girlanda, Messina

V. Grasso, Messina

Ch. Meroni, Milan

F. Neri, Messina

V. Pirronello, Catania

G. Rossi, Victoria, Australia

V. Russo, Florence

G. Saitta, Messina

S. Valeri, Modena

Physical Section,

Union of Czechoslovak

Mathematicians \& Physicists

L. Bardos, Prague

J. Cirak, Bratislava

J. Horvath, Prague

K. Hricovini, Bratislava

Z. Kluiber, Kladno-Krocehlavy

L. Kraus, Prague

L. Lejcek, Prague

K. Masek, Prague

J. Pastrnak, Prague

J. Petzelt, Prague

L. Skrbek, Rez

P. Streda, Prague

A. Simunek, Prague

V. Smid, Prague

R. Reznicek, Prague
The Netherlands' Physical Society

P.J. Blankert, Amsterdam

V. Brabers, Eersel

N.J. Dam, Arnhem

L. Kamp, Waalre

F. Kniest, Bodegraven

N. Nelson, Hoeven

J.F. van der Veen, Bussum

Polish Physical Society

J. Adamowski, Krakow

J. Chojcan, Wroclaw

P. Ciepielewski, Warsaw

M. Debowska, Wroclaw

E. Debowska, Wroclaw

R. Ewertowski, Wroclaw

H. Figiel, Krakow

J. Holender, Krakow

J. Hoylst, Warsaw

J. Kozlowska, Warsaw

T.M. Krygowski, Warsaw

E. Leja, Krakow

M. Olechowski, Warsaw

A. Ostrasz, Wroclaw

S. Pokorski, Warsaw

M. Praszalowicz, Krakow

A. Roszak, Torun

J. Soltys, Krakow

T. Stapinski, Krakow

J.J. Tatarkiewicz, Warsaw

P. Tomaszewski, Wroclaw

K. Walasek, Szczecin

Portuguese Physical Society

M. Alves Marques, Lisbon

Manuel P. Dos Santos, Braga

L.F.A. Ferreira, Lisbon

E.J.S. Lage, Oporto

Romanian National Committee for Physics

M. Rogalski,

Bucharest-Maguerele

T. Toro, Timisoara
Spanish Royal Society of Physics

Jose Manuel Cabrera, Madrid

Swedish Physical Society

L. Borjesson, Göteborg

W.R. Salaneck, Linköping

R. Scullman, Täby

G. Skarnemark, Göteborg

N.-E. F. Svensson, Järfälla

L. Ward, Uppsala

K. Ziakas, Lund

Swiss Physical Society

H. Drevermann, Geneva

T. Gerber, Borne, NL

A.S. Glass, Zug

P. Günter, Schlieren

K. Jauch, Geneva

H.J. Tiziani, Stuttgart, D

\section{CATEGORY 4d)}

American Physical Society

N. Benczer-Koller,

New Brunswick, NJ

P. Campbell, London, UK

A. Choudry, Amsterdam, NL

M. Dresselhaus, Cambridge, MA

Y. Frishman, Rehovot, ISR

H. Gallagher, Bowie, MD

C. Grabbe, lowa City, IA

Behram. N. Kursunoglu,

Coral Gables, FL

K. Kwiatkowski,

Bloomington, IN

P. O'Shea, College Park, MD

J. Park, Raleigh, NC

Dutch Astronomical Society

E. Brinks, Garching, D

V. Icke, Leiden

\section{ASSOCIATE MEMBERS}

KFKI - Central Research

Institute for Physics

POB 49

H-1525 Budapest 114

Institute for Energy Technology

POB 40

$\mathrm{N}-2007 \mathrm{Kjeller}$ 